\title{
OTIMIZAÇÃO DE MULTIPRODUTOS EM POVOAMENTOS FLORESTAIS ${ }^{1}$
}

\author{
Thelma Shirlen Soares ${ }^{2}$, Antonio Bartolomeu do Vale ${ }^{3}$, Helio Garcia Leite ${ }^{3}$ e Carlos Cardoso Machado ${ }^{3}$
}

\begin{abstract}
RESUMO - O objetivo principal deste estudo foi a otimização da colheita de toras, visando a sua conversão em multiprodutos. Para isto, foram utilizados dados provenientes de povoamentos de Eucalyptus grandis, localizados no município de Sabinópolis-MG. Para realização da conversão otimizada, utilizaram-se informações a respeito das alternativas de comercialização da madeira produzida nos povoamentos e das características dos produtos. Foram consideradas três alternativas de uso para as toras: madeira para celulose, energia e serraria, com diferentes dimensões quanto aos diâmetros mínimo e máximo e ao comprimento das toras. O problema inicial foi estabelecer para cada alternativa de uso a combinação otimizada que proporcionasse maior rendimento. Os resultados da otimização demonstraram que os comprimentos de 1,1, 2,0 e 2,2 m (toras para energia, serraria e celulose, respectivamente) proporcionaram maior rendimento volumétrico. Após selecionar o comprimento ótimo da tora para cada alternativa de uso, considerou-se a combinação desses comprimentos a fim de verificar a viabilidade da destinação dos povoamentos para multiprodutos. Para constatar se as otimizações realizadas representaram as opções mais lucrativas para o povoamento em estudo, fez-se uma análise financeira da produção, através do método do valor presente líquido (VPL), para verificar se o uso da madeira para multiprodutos é mais lucrativo que a destinação da madeira para um só uso (celulose ou energia ou serraria). Os valores obtidos indicaram que a colheita florestal, visando o uso múltiplo, é mais rentável que o uso único. Assim, os multiprodutos demonstraram ser a melhor alternativa de remuneração do produto advindo de povoamentos florestais.
\end{abstract}

Palavras-chave: Sortimentos, programação dinâmica e madeira.

\section{OPTIMIZATION OF MULTIPRODUCTS IN FOREST STANDS}

\begin{abstract}
This work aimed to optimize log harvesting by converting them into multiproducts. Thus, data originated from Eucalyptus grandis stands, located in Sabinópolis-MG, were used. Optimized conversion was carried out based on information obtained on available commercial alternatives for logs produced in the stands and on product characteristics. Three alternatives were selected for log use cellulose, energy and sawing, with different maximum and minimum diameter and log length dimensions. The initial problem was to establish an optimized combination that would promote the greatest yield for each utilization alternative. Optimization results show that 1.1; 2.0 and $2.2 \mathrm{~m}$ lengths (for energy, sawing and cellulose use, respectively) resulted in greater volumetric production. After selecting the optimum length for each utilization alternative, the combination of these lengths was considered to verify the viability of using the stands for multiproducts. To verify whether optimization was a profitable option an economic analysis of the production was carried out focusing multiproducts versus a single product (cellulose, energy or sawing), by applying the Net Present Value method. The results indicate that multiproduct harvest is more profitable than single use, being the best alternative for forest standproduct profitability.
\end{abstract}

Key words: $\quad$ Assortment, dynamic programming and timber.

1 Recebido para publicação em 21.2.2002. Aceito para publicação em 4.11.2003.

Parte integrante da tese de mestrado (DEF/UFV) do primeiro autor. Bolsista do CNPq-Brasil.

2 Eng. Florestal, Doutoranda em Ciência Florestal, Departamento de Engenharia Florestal da Universidade Federal de Viçosa UFV, 36570-000 Viçosa-MG, <thelsoares@ bol.com.br>; ${ }^{3}$ Professor do Departamento de Engenharia Florestal - UFV. 


\section{INTRODUÇÃO}

A madeira proveniente dos povoamentos de Eucalyptus sp. era, até alguns anos, exclusivamente voltada para produção de matéria-prima para celulose, carvão vegetal, moirões e postes. Porém, com o crescente desenvolvimento e o aumento da demanda de mercado por produtos madeiráveis, as empresas do setor florestal estão buscando ampliar as possibilidades de utilização da madeira e diversificar a produção (Assis, 2000).

A tendência atual é que parte dessas plantações seja utilizada segundo o conceito de florestas para multiprodutos, em que de um mesmo fuste de uma árvore consegue-se extrair madeira para laminação, serraria, fabricação de papel e celulose e, ainda, aproveitar os resíduos da madeira para fabricação de chapas de fibras e geração de energia, entre outros produtos, sendo essa, provavelmente, a opção que apresente maior possibilidade de remuneração do produto advindo de povoamentos florestais.

Nesse contexto, pode-se perceber uma tendência entre as empresas florestais brasileiras de se estruturarem operacional e administrativamente dentro de metodologia gerencial mais moderna, adotando novas tecnologias que permitam seu desenvolvimento e maior retorno financeiro.

Tendo em vista as inúmeras vantagens advindas da conversão de árvores em multiprodutos, estudos têm sido realizados com o objetivo de estabelecer o melhor aproveitamento na conversão de troncos. Em países de clima temperado, a pesquisa operacional é comumente utilizada no planejamento da produção de povoamentos florestais. Contudo, nas florestas tropicais brasileiras, sejam elas nativas ou plantadas, estudos utilizando métodos de pesquisa operacional, principalmente a programação dinâmica, ainda são incipientes, com um número ainda reduzido de experiências. Com relação à utilização da técnica de programação dinâmica, podem ser citados, dentre outros, os trabalhos realizados por Ahrens (1992), Leite et al. (1995a), Lima et al. (1997), Filgueiras (1997), Arce (1999) e Chichorro (2000). Entretanto, até o momento não se tem conhecimento, na literatura brasileira, da utilização da programação dinâmica na definição do tamanho ideal de toras a serem colhidas em povoamentos florestais. Diante do exposto, considerando a necessidade de maior eficiência na utilização dos recursos florestais, realizou-se o presente estudo cujo objetivo foi a otimização da colheita de toras

R. Árvore, Viçosa-MG, v.27, n.6, p.811-820, 2003 de eucalipto, visando a conversão em multiprodutos.

\section{MATERIAL E MÉTODOS}

\section{1. Área de Estudo e Fonte de Dados}

Foram utilizados dados obtidos de 33 parcelas permanentes (área amostral igual a $1.506,70 \mathrm{~m}^{2}$ ), provenientes do inventário florestal contínuo (IFC) de povoamentos de Eucalyptus grandis, localizados no município de Sabinópolis-MG, cuja medição foi efetuada na idade de 9 anos.

Em cada parcela foram registrados os diâmetros a 1,3 $\mathrm{m}$ de altura do solo (dap) de todas as árvores e a altura total $(H t)$ de algumas árvores. Para obtenção do volume real, procedeu-se à cubagem de 84 árvoresamostras, em diferentes classes de diâmetro. Essas informações foram utilizadas na determinação da distribuição diamétrica e no ajuste de modelos para estimativa da produção do povoamento.

\subsection{Modelos Hipsométrico, Volumétrico e de Taper}

Para estimar o estoque de colheita do povoamento foram ajustados, individualmente, os modelos hipsométrico, volumétrico e de taper. A altura total das árvores foi obtida através do modelo hipsométrico de Campos et al. (1986), citados por Gomes (1996). Para obtenção do volume por árvore, optou-se pela utilização do modelo de múltiplos volumes proposto por Leite et al. (1995b), que é capaz de estimar simultaneamente, de maneira precisa e consistente, o volume total e o volume comercial para qualquer diâmetro com e, ou, sem casca. Já para quantificar os multiprodutos e os volumes de partes do fuste, de acordo com as características estabelecidas, quanto ao comprimento das toras e aos diâmetros máximo e mínimo, ajustou-se o modelo de Demaerschalk, modificado por Leite \& Garcia (2001). A relação funcional dos modelos ajustados é dada por:

Modelo hipsométrico:

$\ln H t=\beta_{0}+\beta_{1}\left(\frac{1}{d a p}\right)+\beta_{2} \ln (H d)+\ln \left(\varepsilon_{i}\right)$

Modelo volumétrico:

$V=\beta_{0} d a p^{\beta_{1}} H t^{\beta_{2}}\left[e^{-\beta_{3}(T x / d a p)}\right]\left(1-\left(\frac{d}{d a p}\right)^{1+\beta_{4} d}\right)+\varepsilon_{i}$ 
Modelo de taper:

$$
\left(\frac{d_{i}}{d a p}\right)^{2}=10^{2 \beta_{0}} d a p^{\left(2 \beta_{1}-2\right)} H t^{2 \beta_{2}}\left(H t-h_{i}\right)^{2 \beta_{3}} e^{\left(\beta_{4} \frac{T x}{d a p}\right)}+\varepsilon_{i}
$$

em que $d a p=$ diâmetro medido a 1,3 $\mathrm{m}$ de altura do solo $(\mathrm{cm}) ; d=$ diâmetro comercial ou superior $(\mathrm{cm}) ; d_{i}=$ diâmetro comercial na altura $\mathrm{h}_{\mathrm{i}}(\mathrm{cm}) ; H t=$ altura total (m); $H d=$ altura dominante $(\mathrm{m}) ; h_{i}=$ altura onde ocorre o diâmetro comercial $\mathrm{d}_{\mathrm{i}}(\mathrm{m}) ; V=$ volume $\left(\mathrm{m}^{3}\right) ; \mathrm{ln}=$ logaritmo neperiano; $\mathrm{e}=$ base dos logaritmos neperianos; $T x=$ variável binária ( 0 para volume ou diâmetro $d_{i}$ com casca e 1 para volume ou diâmetro $d_{i}$ sem casca); $\beta_{\mathrm{i}}=$ parâmetro a ser estimado $(\mathrm{i}=0,1, \ldots, 4)$; e $\varepsilon_{\mathrm{i}}=$ erro aleatório, $\varepsilon_{\mathrm{i}} \sim \operatorname{NID}\left(0, \sigma^{2}\right)$.

Após o ajuste dos modelos, procedeu-se à sua validação a fim de verificar a existência ou não de tendenciosidade nos ajustes realizados. Para tal, optou-se pelo uso do teste de L\&O, proposto por Leite \& Oliveira (2002).

O teste de L\&O caracteriza-se por ser um procedimento estatístico para avaliar a identidade de métodos analíticos, cujo procedimento resulta da combinação do teste de $\mathrm{F}\left(\mathrm{FH}_{0}\right)$ de Graybill, do teste t para erro-médio $\left(t_{\bar{e}}\right)$ e da análise de correlação linear $\left(r_{Y_{j} Y_{1}}\right)$ entre os valores observados $\left(Y_{j}\right)$ e os valores estimados $\left(Y_{1}\right)$. $\mathrm{O}$ teste compara o valor observado, como variável dependente, e o valor estimado, como variável independente. Assim, para situações em que $\mathrm{F}\left(\mathrm{H}_{0}\right)$ e $t_{\bar{e}}$ forem nãosignificativos (ns) e $r_{Y_{j} Y_{1}} \geq 1-|\bar{e}|$, conclui-se que os valores estimados pela regressão não diferem dos valores observados.

\subsection{Alternativas de Conversão da Madeira e Comércio dos Multiprodutos}

Uma das informações necessárias em modelos de otimização é a definição do melhor uso da madeira, o que garantirá o melhor aproveitamento das árvores. Essa definição deve ser feita, levando-se em consideração informações como qualidade e dimensões do fuste. Deste modo, o tronco de uma árvore pode ser subdividido em toras e estas podem ter diferentes destinos, proporcionando maior rentabilidade volumétrica e econômica (Chichorro, 2000).
Foram consideradas três alternativas de conversão dos troncos da madeira em toras: madeira para celulose, energia e serraria. As especificações das dimensões dos diâmetros mínimo (dmin) e máximo (dmax) com casca estão apresentadas na Figura 1. As informações referentes aos custos e às receitas da madeira comercializada, por alternativa de uso, foram obtidas nas principais empresas do setor e estão apresentadas no Quadro 1.

Neste estudo, considerou-se que a venda da produção é feita de acordo com a demanda. Para tanto, admitiuse que qualquer quantidade e tipo de tora produzida será comercializada, isto é, que existe demanda e não há restrição de quantidade.

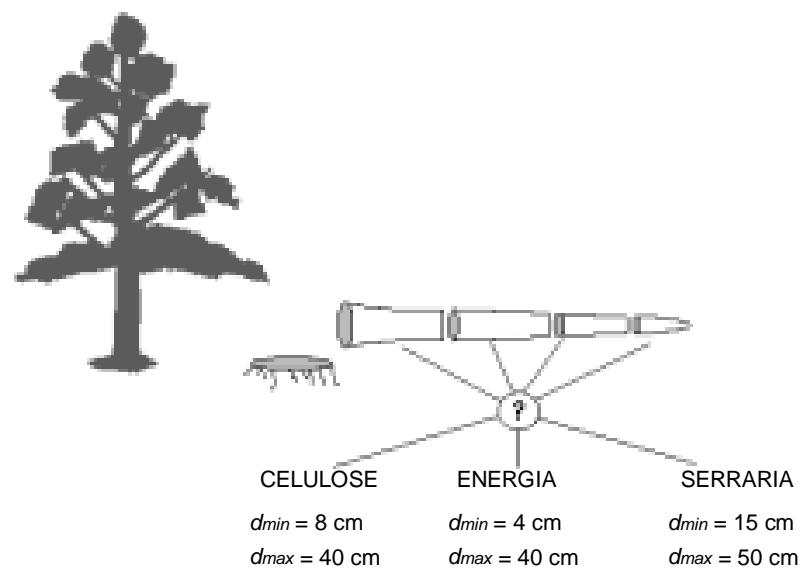

Fonte: Adaptado de Oliveira \& Oliveira (2000).

Figura 1 - Segmentação do fuste em função das alternativas de uso, representando os diâmetros máximo $\left(d_{m q x}\right)$ e mínimo $\left(d_{\min }\right)$ para as toras em função das alternativas de uso.

Figure 1 - $f$ Segmentation of the bole in function of the use alternatives, representing the maximum ( $\mathrm{d}$ ) and minimum diameters $\left(\mathrm{d}_{\text {min }}\right)$ for the logs for use alternatives (Source: Adapted from Oliveira \& Oliveira, 2000).

Quadro 1 - Valor comercial das toras, de acordo com as alternativas de uso

Table 1 - Commercial value of logs, according to use alternatives

\begin{tabular}{|l|c|c|}
\hline Uso da Tora & Preço $\left(\mathrm{US} \$ / \mathrm{m}^{3}\right)^{*}$ & Custo $\left(\mathrm{US} \$ / \mathrm{m}^{3}\right)^{*}$ \\
\hline Celulose & 11,50 & 6,88 \\
\hline Energia & 3,41 & 2,04 \\
\hline Serraria & 13,43 & 8,04 \\
\hline
\end{tabular}

* Informações referentes ao mês de abril de 2001, sendo US $\$ 1,00=$ $\mathrm{R} \$ 2,50$.

R. Árvore, Viçosa-MG, v.27, n.6, p.811-820, 2003 
Em uma primeira etapa, foram selecionadas três alternativas de uso da madeira: toras para celulose, energia e serraria. Foram considerados diferentes comprimentos de tora (Figura 2), com base nos comprimentos utilizados pelas principais empresas do setor florestal. Desta forma, a otimização do sortimento foi composta de três casos: otimizar os comprimentos de colheita das toras para celulose, energia e serraria, respectivamente, visando selecionar a alternativa que proporcionasse maior rendimento volumétrico.

Em uma segunda etapa, os comprimentos selecionados foram agrupados em uma alternativa de otimização denominada de multiprodutos, a fim de estabelecer uma combinação ótima para o povoamento em estudo.

\subsection{Método de Otimização}

A otimização da conversão das toras em multiprodutos foi realizada através do método de programação dinâmica (PD). Após a obtenção dos dados e das informações de distribuição diamétrica, das equações de altura, volume e taper e das características dos produtos, foram realizadas simulações que permitiram otimizar os multiprodutos do povoamento em estudo.

Para efetuar essas simulações, foi desenvolvido, através do Turbo Pascal 7.0, um modelo de otimização denominado Bartho, adaptado a partir dos modelos desenvolvidos por Leite (1994) e Lima (1996). A função de recorrência utilizada na conversão (Figura 3 ) permite solucionar a seguinte situação: "dado um tronco de comprimento $L$ com diâmetro na base $D$ e no topo $d$, como convertê-lo em toras, segundo diferentes usos potenciais, de modo que o retorno econômico, pela conversão de todo o fuste, seja o máximo possível?".

As variáveis do modelo de PD, representado pela função de recorrência, foram definidas como:

$\mathrm{F}_{\mathrm{nz}}(\mathrm{Ht}, \mathrm{D}, \mathrm{Hb})=$ função a ser otimizada;

$\mathrm{R}_{\mathrm{u}=}$ retorno econômico pela conversão da tora, sendo expresso por

$\sum_{\mathrm{w}=1}^{\mathrm{w}} \mathrm{Q}_{\text {iuw }}\left(\mathrm{jz.kz}, \mathrm{dmin}_{\mathrm{i}}, \mathrm{dmax}_{\mathrm{i}}\right) \times \mathrm{P}_{\text {iuw }}-\sum_{\mathrm{w}=1}^{\mathrm{w}} \sum_{\mathrm{y}=1}^{\mathrm{y}} \mathrm{C}_{\text {yiuw }}\left(\mathrm{jz.kz}, \mathrm{dmin}_{\mathrm{i}}, \mathrm{dmax}_{\mathrm{i}}\right) ;$

$\mathrm{f}_{\text {nz-jz.kz }}=$ função a ser otimizada no estado anterior;

$\mathrm{Q}_{\text {iuw }}=$ volume total do produto final;

$\mathrm{P}_{\text {iuw }}=$ preço do produto final;

$\mathrm{C}_{\text {yiuw }}=$ custo do produto final;

R. Árvore, Viçosa-MG, v.27, n.6, p.811-820, 2003
$\mathrm{F}_{\mathrm{nx}}(\mathrm{nxkx})=$ valor máximo da seção da tora entre os estágios 0 e nx, com intervalos kx no nível 2;

$\mathrm{G}_{\mathrm{ny}}[$ ny.ky, nxkx, nxkx-lx(z)] = máximo valor da função para a espessura definida entre os estágios nx e nxlx(z), como função da variável de estado nyky, no nível 3 ;

$\mathrm{G}_{\mathrm{nx}}[\mathrm{ny} \cdot \mathrm{ky}, \mathrm{nxkx}, \mathrm{nxkx}-\mathrm{lx}(\mathrm{z})]$ = máximo valor no estágio ny da seção da tora, entre os estágios nx e nx-lx(z) no nível 2 e entre os estágios 0 e ny no nível 3;

$\mathrm{V}\{\mathrm{w}, \operatorname{nxkx}, \operatorname{nyky}, \mathrm{lx}(\mathrm{z}), \mathrm{ly}(\mathrm{z})\}=$ valor do produto $\mathrm{w}$, obtido nas coordenadas nxkx e nyky, de espessura lx(z) e de largura ly(z);

$\mathrm{Ht}=$ altura total da árvore $(\mathrm{m})$;

$\mathrm{Hb}=$ altura do toco remanescente $(\mathrm{m})$;

$\mathrm{D}=$ diâmetro com casca a 1,30 m de altura do solo $(\mathrm{cm})$;

$1 \mathrm{x}(\mathrm{z})$ = variável decisória no nível 2 , correspondente à largura a ser cortada entre dois estágios, consecutivos ou não;

ly $(\mathrm{z})$ = variável decisória no nível 3, correspondente à largura a ser cortada entre dois estágios, consecutivos ou não;

nx = número de estágios contidos na parte comercial no tronco, no nível 2;

ny = número de estágios contidos na parte comercial no tronco, no nível 3;

$n \times k x=$ variável de estado no nível 2;

nyky = variável de estado no nível 3;

$\mathrm{z}=$ número total de produtos;

jz $=$ comprimento da tora $(2 \leq \mathrm{jz} \leq \mathrm{ht}$, em que $2 \mathrm{~m}$ é o comprimento mínimo da tora);

$\mathrm{kz}=$ intervalo de comprimento no eixo $\mathrm{z}$, ao longo do tronco $(\mathrm{m})$;

skx $=$ espessura do corte no nível 2 ;

sky = espessura do corte no nível 3;

$\mathrm{dmin}_{\mathrm{i}}=$ menor diâmetro da tora analisada $(\mathrm{cm})$;

$\operatorname{dmax}_{\mathrm{i}}=$ maior diâmetro da tora analisada $(\mathrm{cm})$;

$\mathrm{j}$ = número de árvores ou classes diamétricas;

$\mathrm{u}=$ refere-se aos diferentes usos a que a tora pode ser destinada;

$\mathrm{w}=$ produto final proveniente da tora $\mathrm{i}$, convertida no uso u; e

$\mathrm{y}=$ refere-se aos tipos de custos operacionais que envolvem o processo de toragem. 


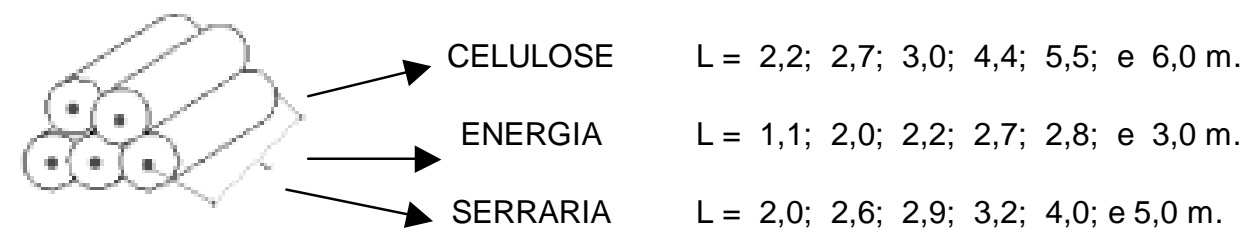

Figura 2 - Comprimentos de tora (L) avaliados, para cada alternativa de uso, no modelo de otimização. Figura 2 - Log lengths $(L)$ evaluated for each use alternative under the otimization model.

Algoritmo BARTHO

Nível 1: Conversão dos troncos em toras:

$$
F_{n z}(H t, D, H b)=\underset{j z, u}{\operatorname{Max}}\left\{R_{u}\left(j z . k z, d \min _{i}, \operatorname{dmax}_{i}\right)+f_{n z-j z . k z}(n z \cdot k z-j z . k z, D, H b)\right\}
$$

Nível 2: Conversão de toras em produtos finais (corte eixo $x$ )

$$
F_{n x}(n x . k x)=\operatorname{Max}_{z \in Z}\left\{G_{n y}[n y \cdot k y, n x k x, n x k x-l x(z)]+F_{n x-l x(z) / k x}(n x k x-l x(z)-s k x)\right\}
$$

Nível 3: Conversão de toras em produtos finais (corte eixo y)

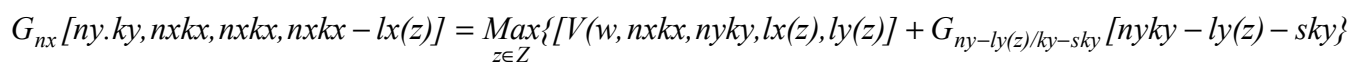

Figura 3 - Relação de recorrência do modelo de PD (Adaptado de Leite et al., 1995a e Lima et al., 1997).

Figure 3 - PD model recurrence relation (Adapted from: Leite et al., 1995a and Lima et al., 1997).

O aplicativo Bartho consta da manipulação de arquivos de dados e otimização pelo modelo de programação dinâmica. Assim, a partir das características das árvores (comprimento e diâmetros máximo e mínimo das toras) e do preço de venda de cada alternativa de uso, o algoritmo fornece a conversão das árvores em toras, de acordo com sua posição no tronco e a produção de madeira por uso otimizado, considerando as alternativas de uso com as respectivas combinações, volume total com casca (VTCC/ha) e volume total sem casca (VTSC/ha), de acordo com a característica do uso.

\subsection{Avaliação Financeira}

A avaliação financeira consiste na verificação da viabilidade de determinada atividade. Neste estudo a análise foi realizada com o método do valor presente líquido (VPL), que tem como finalidade determinar um valor no instante considerado inicial, a partir de um fluxo de caixa formado por uma série de receitas e despesas.
O VPL de um projeto de investimento pode ser definido como a soma algébrica dos valores descontados do fluxo de caixa a ele associado. Conceitualmente, a viabilidade financeira de um projeto analisado por este método é indicada pela diferença positiva entre receitas e custos, atualizados à determinada taxa de juros (Rezende \& Oliveira, 1993).

Para as análises financeiras, foram utilizados dados de custos de condução e colheita do povoamento, juntamente com dados de preços de venda da madeira, definindo-se uma planilha de custos e receitas. Os custos referentes às operações de implantação, manutenção anual e colheita florestal estão apresentados no Quadro 3. Como custo da terra definiu-se o valor de US\$ 500,00/ha. O transporte da madeira foi considerado ser de responsabilidade do comprador. Os preços de venda utilizados equivalem às receitas apresentadas no Quadro 1. Cabe ressaltar que foram considerados os custos apresentados no Quadro 2 para todas as alternativas analisadas.

R. Árvore, Viçosa-MG, v.27, n.6, p.811-820, 2003 
Quadro 2 - Dados de custos de produção de madeira considerando o uso para celulose e o uso de multiprodutos

Table 2 - Data of timber production costs for cellulose use or multiproducts use

\begin{tabular}{|l|c|c|}
\hline \multicolumn{1}{|c|}{ Atividade } & $\begin{array}{c}\text { Ano de } \\
\text { Ocorrência }\end{array}$ & $\begin{array}{c}\text { Custos } \\
\text { (US } \$ / \mathrm{ha})\end{array}$ \\
\hline Implantação* & 0 & 500,00 \\
\hline Capina & 1 & 40,00 \\
\hline Capina & 2 & 18,00 \\
\hline Manutenção & 1 a 9 & 10,00 \\
\hline Colheita** & 9 & 6,00 \\
\hline Administração & anual & $10 \%$ do custo total \\
\hline
\end{tabular}

* Os custos de implantação envolvem as operações de infra-estrutura, construção de estradas e aceiros, desmatamento, destoca, limpeza da área, preparo do terreno, plantio e replantio de mudas, combate às formigas e aplicação de fertilizantes.

** Custos expressos em US $\$ / \mathrm{m}^{3}$.

Quadro 3 - Distribuição diamétrica do povoamento Table 3 - Stand diameter distribution

\begin{tabular}{|c|c|c|c|}
\hline $\begin{array}{c}\text { Valor Central da } \\
\text { Classe Diamétrica } \\
(\mathrm{cm})\end{array}$ & $\begin{array}{l}\text { Altura Total } \\
\text { Média (m) }\end{array}$ & $\begin{array}{c}\text { Freqüência } \\
\left(n^{\circ} \text { de árv./ha) }\right.\end{array}$ & $\begin{array}{c}\text { Área Basal } \\
\text { Média } \\
\left(\mathrm{m}^{2} / \mathrm{ha}\right) \\
\end{array}$ \\
\hline 5,0 & 10,1 & 29 & 0,0560 \\
\hline 7,0 & 14,1 & 84 & 0,3240 \\
\hline 9,0 & 18,2 & 117 & 0,7422 \\
\hline 11,0 & 21,5 & 135 & 1,2787 \\
\hline 13,0 & 23,7 & 164 & 2,1731 \\
\hline 15,0 & 26,0 & 169 & 2,9869 \\
\hline 17,0 & 27,7 & 169 & 3,8365 \\
\hline 19,0 & 29,2 & 139 & 3,9278 \\
\hline 21,0 & 30,6 & 131 & 4,5227 \\
\hline 23,0 & 31,6 & 89 & 3,6903 \\
\hline 25,0 & 33,1 & 78 & 3,8068 \\
\hline 27,0 & 34,1 & 42 & 2,3909 \\
\hline 29,0 & 35,2 & 22 & 1,4448 \\
\hline 31,0 & 36,2 & 9 & 0,6504 \\
\hline 33,0 & 38,0 & 5 & 0,3968 \\
\hline 35,0 & 38,1 & 4 & 0,3826 \\
\hline 37,0 & - & - & - \\
\hline 39,0 & 42,0 & 1 & 0,0792 \\
\hline Total/Média & & 1.387 & 1,8161 \\
\hline
\end{tabular}

Neste estudo, comparou-se a rentabilidade dos multiprodutos (combinação dos comprimentos selecionados para celulose, energia e serraria) com a rentabilidade

R. Árvore, Viçosa-MG, v.27, n.6, p.811-820, 2003 proporcionada pela destinação da madeira do povoamento a um único uso (celulose ou energia ou serraria).

Com relação às taxas de desconto, considerou-se a aplicação de taxas reais de desconto de 6, 9 e $12 \%$ ao ano, no cálculo do VPL. Essas taxas foram escolhidas por abrangerem os níveis mais usados pelo setor florestal (Oliveira et al., 1998).

\section{RESULTADOS E DISCUSSÃO}

\subsection{Distribuição Diamétrica do Povoamento}

As medições diamétricas, agrupadas em classes de 2,0 cm de amplitude, estão apresentadas no Quadro 3. Essa distribuição, juntamente com os dados do Quadro 1, foi utilizada para aplicação do modelo de otimização.

\subsection{Equações Hipsométrica, Volumétricas e de Taper}

As estimativas dos parâmetros e das medidas de precisão das equações obtidas estão apresentadas no Quadro 4.

Os resultados da validação das equações, realizada pelo teste de L\&O, estão no Quadro 5, onde é possível verificar que todas as equações obtidas no ajuste apresentaram estimativas precisas na predição das variáveis.

\subsection{Otimização da Produção}

Os resultados da otimização, considerando a conversão da tora em usos individuais (madeira para celulose, energia e serraria), encontram-se no Quadro 6.

Analisando conjuntamente os resultados obtidos na conversão das toras para celulose, energia e serraria observa-se que, em todos os casos, os menores comprimentos propiciaram maior rendimento volumétrico.

Após realizar a otimização da conversão para cada uso de forma separada, selecionou-se, para cada alternativa de uso, o comprimento que proporcionou o maior retorno volumétrico, ou seja, 2,2 m para celulose, 1,1 m para energia e $2,0 \mathrm{~m}$ para serraria. As alternativas de comprimento selecionadas foram então agrupadas, a fim de obter a otimização das alternativas de uso da madeira de forma integrada para todo o povoamento. Os resultados da otimização que definiu a melhor opção para o povoamento estão apresentados no Quadro 7. 
Quadro 4 - Parâmetros e medidas de precisão para as equações ajustadas Table 4 - Parameters and precision measures for the equations fitted

\begin{tabular}{|l|c|c|c|c|c|c|c|}
\hline \multicolumn{1}{|c|}{ Equação } & $\beta_{0}$ & $\beta_{1}$ & $\beta_{2}$ & $\beta_{3}$ & $\beta_{4}$ & $R^{2}$ & $S y x$ \\
\hline Hipsométrica & 2,0165 & $-7,9756$ & 0,5076 & - & - & 0,9485 & 0,0858 \\
\hline Volumétrica & 0,000057 & 1,6884 & 1,1399 & $-3,0295$ & 0,2494 & 0,9958 & 0,0486 \\
\hline Taper & 0,0420 & 0,9021 & $-0,6842$ & 0,7574 & $-2,7317$ & 0,9780 & 0,0724 \\
\hline
\end{tabular}

Quadro 5 - Resultados do teste de L\&O para validação das equações ajustadas, para um nível de significância de 5\% Table 5 - Results of the L\&O test results for validation of the adjusted equations, at $5 \%$ probability

\begin{tabular}{|l|c|c|c|l|}
\hline \multicolumn{1}{|c|}{ Equação } & $\mathrm{F}\left(\mathrm{H}_{0}\right)$ & $\mathrm{t} \bar{e}$ & $r_{Y_{j} Y_{1}}$ & \multicolumn{1}{c|}{ Conclusão } \\
\hline Hipsométrica & $0,003 \mathrm{~ns}$ & $0,850 \mathrm{~ns}$ & 0,974 & $\begin{array}{l}Y_{\mathrm{j}}^{*} \text { é estatisticamente igual a } Y_{1}^{* * *} \text {, para o nível de } \\
\text { significância estabelecido. }\end{array}$ \\
\hline Volumétrica & $0,128 \mathrm{~ns}$ & $1,932 \mathrm{~ns}$ & 0,925 & $\begin{array}{l}Y_{\mathrm{j}}^{*} \text { é estatisticamente igual a } Y_{1}^{* * *}, \text { para o nível de } \\
\text { significância estabelecido. }\end{array}$ \\
\hline Taper & $0,100 \mathrm{~ns}$ & $1,956 \mathrm{~ns}$ & 0,463 & $\begin{array}{l}Y_{\mathrm{j}}^{*} \text { é estatisticamente igual a } Y_{1}^{* *} \text {, para o nível de } \\
\text { significância estabelecido. }\end{array}$ \\
\hline
\end{tabular}

$* Y_{\mathrm{j}}=$ valores observados $\mathrm{e} * * Y_{1}=$ valores estimados.

Quadro 6 - Volume total com casca (VTCC) e sem casca (VTSC) obtidos da otimização do tamanho das toras para celulose, energia e serraria

Table 6 - Total volume with bark (VTCC) and without bark (VTSC) obtained from optimization of $10 \mathrm{~g}$ size for cellulose, energy and sawing

\begin{tabular}{|c|c|c|c|}
\hline Uso & $\begin{array}{c}\text { Comprimento } \\
(\mathrm{m})\end{array}$ & $\begin{array}{l}\text { VTCC } \\
\left(\mathrm{m}^{3} / \mathrm{ha}\right)\end{array}$ & $\begin{array}{l}\text { VTSC } \\
\left(\mathrm{m}^{3} / \mathrm{ha}\right)\end{array}$ \\
\hline \multirow{6}{*}{ Celulose } & 2,2 & 378,576 & 331,595 \\
\hline & 2,7 & 377,130 & 330,882 \\
\hline & 3,0 & 369,817 & 324,722 \\
\hline & 4,4 & 363,575 & 319,231 \\
\hline & 5,5 & 356,056 & 312,936 \\
\hline & 6,0 & 354,304 & 311,442 \\
\hline \multirow{6}{*}{ Energia } & 1,1 & 422,393 & 367,677 \\
\hline & 2,0 & 421,238 & 366,681 \\
\hline & 2,2 & 420,925 & 366,451 \\
\hline & 2,7 & 420,092 & 365,797 \\
\hline & 2,8 & 420,924 & 366,444 \\
\hline & 3,0 & 421,270 & 366,757 \\
\hline \multirow{6}{*}{ Serraria } & 2,0 & 208,568 & 186,320 \\
\hline & 2,6 & 208,112 & 185,910 \\
\hline & 2,9 & 201,888 & 180,388 \\
\hline & 3,2 & 204,779 & 182,849 \\
\hline & 4,0 & 189,911 & 169,876 \\
\hline & 5,0 & 199,302 & 178,097 \\
\hline
\end{tabular}

Quadro 7 - Volume total com casca (VTCC) e sem casca (VTSC) obtidos da otimização do tamanho das toras para multiprodutos

Table 7 - Total volume with bark (VTCC) and without bark (VTSC) obtained from optimization of log size for multiproducts

\begin{tabular}{|c|c|c|}
\hline Uso & VTCC $\left(\mathrm{m}^{3} / \mathrm{ha}\right)$ & VTSC $\left(\mathrm{m}^{3} / \mathrm{ha}\right)$ \\
\hline Celulose & 165,268 & 141,337 \\
\hline Energia & 40,525 & 33,201 \\
\hline Serraria & 216,723 & 193,201 \\
\hline Total & 422,516 & 367,738 \\
\hline
\end{tabular}

Pelos resultados obtidos na otimização da combinação dos usos, obteve-se a receita total de cada alternativa e combinação de uso otimizado. Para obtenção do peso total de cada alternativa de uso, considerou-se a densidade básica média do Eucalyptus grandis igual a $570 \mathrm{~kg} / \mathrm{m}^{3}$, conforme estudo de Carmo (1996), a qual proporcionou um fator de conversão de $1,7544 \mathrm{~m}^{3}$ para cada tonelada de madeira obtida. Então, a produção em metro cúbico foi convertida para tonelada, possibilitando obter a receita total de cada alternativa de uso otimizado (Quadro 8). Para obter a receita líquida da otimização, foram considerados os custos e as receitas apresentados no Quadro 1.

R. Árvore, Viçosa-MG, v.27, n.6, p.811-820, 2003 
Quadro 8 - Produção e receita por alternativa de uso Table 8 - Production and revenue per use alternative

\begin{tabular}{|c|c|c|}
\hline Uso & VTCC (t/ha) & Receita (US\$/ha) \\
\hline Celulose & 94,202 & 763,54 \\
\hline Energia & 23,099 & 55,52 \\
\hline Serraria & 123,531 & $1.168,14$ \\
\hline Total & 240,832 & $1.987,19$ \\
\hline
\end{tabular}

A colheita das toras, visando o seu uso múltiplo, comprova, neste caso, a vantagem do manejo de um povoamento florestal destinado a multiprodutos, ou seja, ao destinar cada parte da tora a seu uso mais rentável a empresa atinge o máximo rendimento (através do sortimento), haja vista que a madeira serrada possui um valor agregado maior que o da madeira para celulose ou energia. A destinação do povoamento a multiprodutos apresenta-se, portanto, como a melhor opção de remuneração do produto, uma vez que haverá uma diminuição no risco de perda, decorrente da redução do preço de venda desse ou daquele produto.

\subsection{Avaliação Financeira}

Para constatar se a otimização realizada para os multiprodutos representa realmente a opção mais lucrativa para o povoamento em estudo, realizou-se a verificação da viabilidade financeira dos multiprodutos (celulose + energia + serraria) em comparação com o uso da madeira para apenas uma finalidade (celulose ou energia ou serraria).

Dessa forma, conforme já relatado, foi utilizado o método do VPL para analisar a viabilidade financeira, tendo o investimento sido considerado viável para os casos em que o VPL foi positivo.

Constata-se, ao analisar o Quadro 9, que a alternativa de multiprodutos apresentou-se, do ponto de vista econômico, a mais viável, seguida das opções serraria e celulose. Considerando o uso da madeira para energia observa-se que, para todas as taxas de desconto, a alternativa apresentou-se inviável economicamente, uma vez que apresentou VPL negativo. Um dos aspectos que podem ter prejudicado a viabilidade financeira das toras para energia é o baixo preço considerado, por ocasião deste estudo, para a venda da madeira.

De modo geral, verifica-se que à medida que a taxa de desconto aumenta o risco do empreendimento também

R. Árvore, Viçosa-MG, v.27, n.6, p.811-820, 2003 é maior e o VPL cresce em termos negativos, ou seja, o prejuízo aumenta. Esta sensibilidade às variações na taxa real de desconto usada era esperada, uma vez que altas taxas de desconto tendem a inviabilizar investimentos a longo prazo, devido ao longo tempo de conversão do investimento, como é o caso de investimentos no setor florestal.

Os valores obtidos para o VPL indicam que a alternativa de uso da madeira para um único uso não é recomendável do ponto de vista econômico, no referente caso, em relação ao uso de madeira para multiprodutos (celulose + energia + serraria). Ao colher a madeira somente para celulose, com comprimento da tora igual a $5,5 \mathrm{~m}$, a empresa reduz o aproveitamento da tora.

Para o povoamento em estudo, observa-se que ocorre geração de menor quantidade de resíduos quando da destinação da produção para apenas um uso em relação aos multiprodutos. A diferença do uso único comparado aos multiprodutos é da ordem de 10,40, 0,03 e 50,64\%, para celulose, energia e serraria, respectivamente. Em termos porcentuais, a diferença apresentada pelos usos celulose e energia pode parecer não-significativa. Porém, considerando que a área total florestada de uma empresa e o volume de madeira produzida representam valores consideráveis, verifica-se que ao destinar sua produção apenas para celulose a empresa está perdendo retornos financeiros.

Com a otimização das toras visando o uso para celulose, obteve-se, após a otimização, o comprimento de 2,2 $\mathrm{m}$ como aquele que proporcionou maior rendimento volumétrico.

A produção volumétrica proporcionada pelo corte das toras no comprimento recomendado como ótimo

Quadro 9 - Volume e valor presente líquido (VPL) para um único uso e para multiprodutos, para diferentes taxas de desconto

Table 9 - Volume and net present value (VPL) for single use and for multiproducts use for different discount rates

\begin{tabular}{|l|c|c|c|c|}
\hline \multirow{2}{*}{ Uso } & \multirow{2}{*}{$\begin{array}{c}\text { Volume } \\
\left(\mathrm{m}^{3}\right)\end{array}$} & \multicolumn{3}{|c|}{ VPL (US\$/ha) } \\
\cline { 3 - 5 } & & $6 \%$ a.a & $9 \%$ a.a & $12 \%$ a.a \\
\hline Celulose & 165,268 & 731,05 & 411,79 & 160,70 \\
\hline Energia & 40,525 & $-1280,67$ & $-1199,40$ & $-1137,79$ \\
\hline Serraria & 216,723 & 470,49 & 211,20 & 6,50 \\
\hline Multiprodutos & 422,516 & 920,41 & 561,23 & 279,09 \\
\hline
\end{tabular}


neste estudo (no caso, $2,2 \mathrm{~m}$ ) foi de $378,576 \mathrm{~m}^{3} / \mathrm{ha}$, enquanto a produção do povoamento cujas toras foram cortadas com $6,0 \mathrm{~m}$ foi de $361,665 \mathrm{~m}^{3} / \mathrm{ha}$. É possível observar uma vantagem de $4,5 \%$ no volume propiciado pelas toras de menor comprimento.

Com relação às diferenças monetárias, utilizando o método do VPL obtêm-se valores de US $\$ 411,79 /$ ha e US\$ 371,75/ha para os comprimentos de 2,2 e 6,0 m, respectivamente. Estes números demonstram que, atualmente, as empresas que colhem as toras com $6,0 \mathrm{~m}$ de comprimento estão tendo uma perda, por hectare, da ordem de $10 \%$.

Pode-se observar na Figura 4 que a conversão em toras de menor comprimento propicia melhor aproveitamento do tronco.

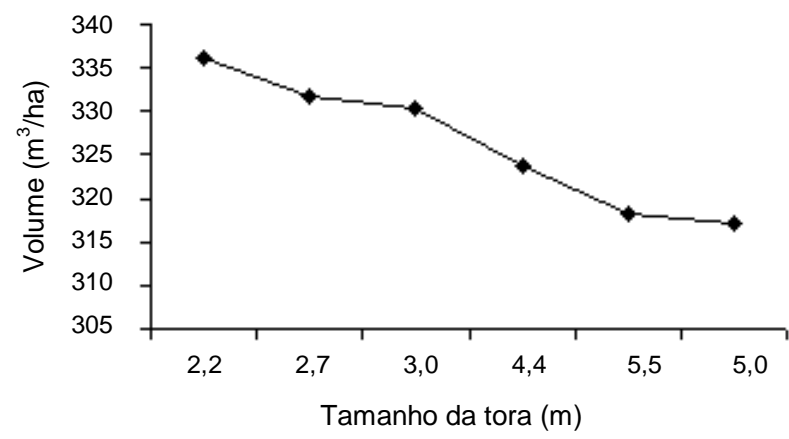

Figura 4 - Comportamento do volume recuperado para diferentes tamanho de tora.

Figure 4-Behavior of the volume recovered volume behavior for different log sizes.

Vale ressaltar que nem sempre as empresas podem operacionalizar a colheita no comprimento indicado como ideal neste estudo, pois, às vezes, elas encontram limitações com relação ao transporte e, ou, comprimento do picador na fábrica. Assim, embora os resultados apontem para uma determinada alternativa otimizada, por motivos técnicos e, ou, operacionais, a empresa poderá ter que destinar sua produção para uma outra alternativa, que, certamente, proporcionará menor retorno econômico.

Portanto, uma aplicação prática desses resultados, em nível de planejamento empresarial, seria utilizar estas informações, extrapolando os resultados para situações semelhantes às utilizadas neste estudo.
Outra opção para a empresa seria utilizar o algoritmo Bartho e processar seus projetos a fim de utilizar as saídas do algoritmo para melhor direcionar o uso ou a comercialização da madeira. A partir dessas informações, os resultados podem ser utilizados de maneira prática e confiável no planejamento e gerenciamento dos povoamentos florestais.

Por fim, cabe ressaltar que em qualquer análise, além do que já foi mencionado, a empresa poderá contemplar outros produtos alternativos e, ou, subprodutos da madeira.

\section{CONCLUSÕES}

De acordo com os resultados obtidos, para as condições em que foram desenvolvidas este estudo, concluiuse que:

- Os melhores tamanhos, sob o critério econômico, para os usos energia, serraria e celulose foram 1,1,2,0 e $2,2 \mathrm{~m}$, respectivamente.

- O emprego do algoritmo BARTHO, por meio da simulação de diferentes cenários, de acordo com as características definidas para cada produto e as condições de mercado, representa uma importante ferramenta de tomada de decisão sobre o uso e comércio dos multiprodutos de povoamentos florestais.

- Os multiprodutos (combinação dos usos celulose, energia e serraria) apresentam maior retorno econômico quando comparado com a destinação da madeira para apenas um uso (celulose ou energia ou serraria).

- As toras de menor comprimento resultam em maiores rendimentos volumétricos devido ao melhor aproveitamento do fuste propiciado por elas, em comparação com comprimentos maiores.

- A implementação de modelos de programação dinâmica, destinados à otimização de multiprodutos, permite aumentar, de forma significativa, o retorno econômico pelo uso da madeira, diminuindo assim o risco do empreendimento.

\section{REFERÊNCIAS BIBLIOGRÁFICAS}

AHRENS, S. A seleção simultânea do ótimo regime de desbastes e da idade de rotação, para povoamentos de Pinus taeda L., através de um modelo de programação dinâmica. 1992. 189 f. Tese (Doutorado em Ciência Florestal) - Universidade Federal do Paraná, Curitiba, 1992. 
ARCE, J. E. Um sistema de programação de transporte de multiprodutos florestais visando a minimização do custo da matéria-prima. 1999. 98 f. Dissertação (Mestrado em Engenharia Florestal) - Universidade Federal do Paraná, Curitiba, 1999.

ASSIS, A. L. Avaliação de modelos polinomiais segmentados e não segmentados na estimativa de diâmetros e volumes comerciais de Pinus taeda. 2000. 198 f. Dissertação (Mestrado em Engenharia Florestal) Universidade Federal de Lavras, Lavras, 2000.

CARMO, A. P. T. Avaliação de algumas propriedades da madeira de seis espécies de eucalipto. $1996.77 \mathrm{f}$. Dissertação (Mestrado em Ciência Florestal) - Universidade Federal de Viçosa, Viçosa.

CHICHORRO, J. F. Análise estrutural e econômica de multiprodutos da madeira. 2000. $241 \mathrm{f}$. Tese (Doutorado em Ciência Florestal) - Universidade Federal de Viçosa, Viçosa, 2000.

FILGUEIRAS, J. F. Um modelo de substituição de equipamentos para minimizar custos operacionais em uma empresa florestal. 1997. 54 f. Tese (Doutorado em Ciência Florestal) - Universidade Federal de Viçosa, Viçosa, 1997.

GOMES, C. R. L. Produção de madeira e carvão em plantações de Eucalyptus camaldulensis e Eucalyptus cloeziana. 1996. 92 f. Dissertação (Mestrado em Ciência Florestal) - Universidade Federal de Viçosa, Viçosa, 1996.

LEITE, H. G. Conversão de troncos em multiprodutos de madeira, utilizando programação dinâmica. 1994. $230 \mathrm{f}$. Tese (Doutorado em Ciência Florestal) - Universidade Federal de Viçosa, Viçosa.

LEITE, H. G.; CAMPOS, J. C. C.; PAULA JUNIOR, G. G. Emprego de um modelo de programação dinâmica para conversão de troncos em multiprodutos da madeira. Revista Árvore, v. 19, n. 4, p. 447-465, 1995a.
LEITE, H. G.; GUIMARÃES, D. P., CAMPOS, J. C. C. Descrição e emprego de um modelo para estimar múltiplos volumes de árvores. Revista Árvore, v. 19, n. 1, p. 65-79, 1995b.

LEITE, H. G.; GARCIA, S. L. R. Pesquisa e desenvolvimentos em inventário, mensurações e manejo florestal na CENIBRA. Viçosa: Sociedade de Investigações Florestais, 2001. 49 p. (Relatório técnico)

LEITE, H. G.; OLIVEIRA, F. H. T. Statistical procedure to test the identity of analytical methods. Communications in soil science plant analysis. New York: v.33, issue 7 e 8, 2002.

LIMA, D. G. Desenvolvimento e aplicação de um modelo de suporte à decisão sobre multiprodutos de povoamentos de eucalipto. 1996. 80 f. Dissertação (Mestrado em Ciência Florestal) - Universidade Federal de Viçosa, Viçosa, 1996.

LIMA, D. G. et al. Um modelo de suporte a decisão sobre multiprodutos de povoamentos de eucalipto. Revista Árvore, v. 21, n. 1, p. 35-48, 1997.

OLIVEIRA, A. D. et al. Avaliação econômica da vegetação de cerrado submetida a diferentes regimes de manejo e de povoamentos de eucalipto plantado em monocultivo.

Revista Cerne, v. 4, n. 1, p. 034-056, 1998.

OLIVEIRA, E. B.; OLIVEIRA, Y. M. M. SISPLAN Sistema para manejo e análise econômica de florestas de pinus. In: CONGRESSO E MOSTRA DE AGRO INFORMÁTICA, 2000, Ponta Grossa. Disponível em: <http://www.infoagro2000.deinfo.uepg.br/artigos/pdf/ info_013.pdf>. Acesso em: 15/08/2001.

REZENDE, J. L. P.; OLIVEIRA, A. D. Avaliação de projetos florestais. Viçosa: Universidade Federal de Viçosa, 1993. 47 p. 